\title{
Effects of Temperature on Styrene Emulsion Polymerization Kinetics
}

\author{
Chorng-Shyan CHERN, Shi-Yow LIN, and Tien-Jung Hsu
}

\author{
Department of Chemical Engineering, National Taiwan University of Science and Technology, \\ 43 Keelung Road, Section 4, Taipei 106, Taiwan
}

(Received November 9, 1998)

\begin{abstract}
The effects of temperature $(T)$ on the kinetics of styrene emulsion polymerization with constant levels of surfactant and initiator were investigated. The production rates of the initiator radicals in water $\left(\rho_{\mathrm{i}}\right)$ increase with $T$, while electrolyte concentrations remain constant. The effect of ionic strength on the particle nucleation and colloidal stability of the latex particles in the course of polymerization can be eliminated. The number of latex particles $\left(N_{\mathrm{p}}\right)$ nucleated is proportional to $\rho_{\mathrm{i}}$ to the 0.27 power, which is not consistent with the Smith-Ewart Case 2 model $\left(N_{\mathrm{p}} \propto \rho_{\mathrm{i}}^{0.4}\right)$. This discrepancy is attributed to the following: (1) particle nuclei are continuously generated beyond the Smith-Ewart Interval I, presumably due to the mixed mode of particle nucleation (micellar nucleation/homogeneous nucleation), (2) the range of the constant polymerization rate region (Smith-Ewart Interval II) becomes narrower when $T$ increases from 50 to $80^{\circ} \mathrm{C}$, (3) the reaction system deviates from Smith-Ewart Case 2 kinetics due to desorption of radicals out of the particles when $T>60^{\circ} \mathrm{C}$, and $(4)$ a significant population of tiny monomer droplets still remains in the reaction mixture beyond the Smith-Ewart Interval II.
\end{abstract} KEY WORDS Emulsion Polymerization / Styrene/Temperature Effect / Kinetics / Particle Nucleation/

Emulsion polymerization involves the propagation of relatively water-insoluble monomer (e.g., styrene (ST)) in numerous submicron latex particles dispersed in an aqueous phase. These latex particles can be stabilized by adsorption of surfactant species (e.g., sodium dodecyl sulfate (SDS)) on the particle surface and polymerization is generally initiated by a water-soluble initiator (e.g., sodium persulfate (SPS)), leading to unique free radical segregation effect. The polymerization is divided into three distinct stages, nucleation of particle nuclei by capture of radicals by monomer-swollen micelles (Interval I), growth of latex particles by recruiting monomer and surfactant from the emulsified monomer droplets (Interval II), and depletion of residual monomer in latex particles (Interval III) according to the pioneering work of Harkins ${ }^{1}$ and Smith and Ewart. ${ }^{2,3}$ The number of latex particles per unit volume $\left(N_{\mathrm{p}}\right)$ produced can be predicted by the Smith-Ewart Case 2 model:

$$
N_{\mathrm{p}}=K\left(\rho_{\mathrm{i}} / \mu\right)^{0.4}\left(a_{\mathrm{s}}[\mathrm{S}]\right)^{0.6}
$$

where $K$ is the proportional constant, $\rho_{\mathrm{i}}=2 f k_{\mathrm{d}}[\mathrm{I}]$ is the production rate of initiator radicals in the aqueous phase, $f$ is the initiator efficiency factor, $k_{\mathrm{d}}$ is the initiator decomposition rate constant, [I] is the initiator concentration, $\mu$ is the constant particle volumetric growth rate, $a_{\mathrm{s}}$ is the polymer particle-water interfacial area occupied by one molecule of surfactant, and $[\mathrm{S}]$ is the surfactant concentration. The constant polymerization rate during Interval II $\left(R_{\mathrm{p}}\right)$ can be calculated as,

$$
R_{\mathrm{p}}=k_{\mathrm{p}}[\mathrm{M}]_{\mathrm{p}}\left(\bar{n} N_{\mathrm{p}} / N_{\mathrm{a}}\right)
$$

where $k_{\mathrm{p}}$ is the propagation rate constant, $[\mathrm{M}]_{\mathrm{p}}$ is the monomer concentration in latex particles, $\bar{n}$ is the average number of radicals per particle, and $N_{\mathrm{a}}$ is Avogadro number. Equation 1 or 2 (i.e., $N_{\mathrm{p}} \propto[\mathrm{I}]^{0.4}[\mathrm{~S}]^{0.6}$ or $R_{\mathrm{p}} \propto[\mathrm{I}]^{0.4}[\mathrm{~S}]^{0.6}$ ) has been widely used to examine the validity of the Smith-Ewart Case 2 model because $\rho_{\mathrm{i}} \propto$ [I] and $R_{\mathrm{p}} \propto N_{\mathrm{p}}$. Reasonable agreement between the Smith-Ewart model and experimental data obtained from the ST emulsion polymerization was observed. Please refer to ref $3-9$. However, electrically charged colloidal particles are quite sensitive to changes in the electrolyte concentration. Increasing [I] to obtain the dependence of $N_{\mathrm{p}}$ on [I] enhances the ionic strength of the aqueous solution. The increase of counterion concentration compresses the electrical double layer around latex particles, thereby greatly reducing the colloidal stability of the latex particles according to the DLVO theory. ${ }^{10,11}$ Flocculation among interactive particles may thus take place in polymerization and lead to reduction in $N_{\mathrm{p}}$. Increasing [I] may enhance particle surface charge density originating from the sulfate end-groups of polymer. In this case, the colloidal stability of the latex particles is improved due to increased electrostatic repulsion force between two approaching particles with the particle surface charge density. Mysels and Princen ${ }^{12}$ showed that the aggregation number of SDS micelles is 62 in water and becomes 142 in the $0.5 \mathrm{M}$ $\mathrm{NaCl}$ aqueous solution. These data imply that, at constant [S], the concentration of micelles should decrease with increasing electrolyte concentration (or $[\mathrm{I}])$. The $\mathrm{pH}$ of the reaction medium in the absence of buffer may decrease significantly with increasing [I] due to the formation of sulfuric acid in the initiation step. This may have influence on the decomposition rate of initiator and colloidal stability. All these factors make the attempt to verify the Smith-Ewart model more difficult.

This work thus uses another approach to study the influence of $\rho_{\mathrm{i}}$ on $N_{\mathrm{p}}$ for the ST emulsion polymerization. In this approach, the concentrations of SDS and SPS are kept constant. Thus, electrolyte concentration remains constant. The value of $\rho_{\mathrm{i}}=2 f k_{\mathrm{d}}$ [I] was varied simply by changing the reaction temperature $(T=50$ $80^{\circ} \mathrm{C}$ ). Increasing $T$ results in an increase in $k_{\mathrm{d}}$. This increases $\rho_{\mathrm{i}}$ provided that $f$ does not change significantly with $T$. The above problems associated with the change of $[\mathrm{I}]$ in determining the exponent $x$ in the relationship $N_{\mathrm{p}} \propto[\mathrm{I}]^{x}$ should thus be eliminated. Emulsion polymerization kinetics are of primary interest to this study. 


\section{EXPERIMENTAL}

\section{Materials}

The chemicals used were styrene (Taiwan Styrene Co.); sodium dodecyl sulfate $(>99 \%$, Fluka); sodium persulfate $(99 \%$, Riedel-de Haen); hydroquinone $(99 \%$, Nacalai Tesque); nitrogen (Ching-Feng-Harng Co.); deionized water (Barnsted, Nanopure Ultrapure Water System, specific conductance $<0.057 \mu \mathrm{S} \mathrm{cm}^{-1}$ ). The monomer was distilled under reduced pressure before use. All other reagents were used as received.

\section{Polymerization Process}

Batch emulsion polymerization was carried out in a $1500-\mathrm{ml}$ jacketed glass reactor equipped with four evenly spaced baffles, a $45^{\circ}$ pitched 6-bladed agitator, a thermometer and reflux condenser. The polymerization procedure is briefly described as follows. First, the mixture including $755 \mathrm{ml}$ water and $1.82 \times 10^{-2} \mathrm{M} \mathrm{SDS}$ based on total water is charged into the reactor, purged with nitrogen to remove the dissolved oxygen, and heated to the polymerization temperature. The monomer $(1.7 \mathrm{M}$ based on total water) is added to the reactor and the resultant emulsion is thoroughly mixed at $400 \mathrm{rpm}$ for $2 \mathrm{~h}$. This is followed by addition of the initiator solution comprising $1.4 \times 10^{-3} \mathrm{M}$ SPS based on total water and $10 \mathrm{ml}$ water into the reactor to start the polymerization. The temperature is controlled within the range of $\pm 0.5^{\circ} \mathrm{C}$ by the cooling jacket connected to a water bath with thermostatic control and the agitation speed is kept constant at $400 \mathrm{rpm}$ throughout the reaction. During polymerization, the latex sample is taken from the reactor, inhibited with a small amount of hydroquinone, and cooled rapidly by an ice-water bath. The total solid content of the latex product is $15 \%$ for complete monomer conversion.

The latex product was filtered through a 40 -mesh $(0.42 \mathrm{~mm})$ screen and a 200 -mesh $(0.074 \mathrm{~mm})$ screen in series to collect the coagulum. Scraps adhering to the agitator, thermometer and reactor wall were collected. The amounts of coagulum for the latex products prepared at various temperatures are nearly nil. This indicates that the stability of the latex particles is satisfactory in the polymerization. Total solid content was determined by the gravimetric method. Particle size and particle size distribution of the latex sample were determined by transmission electron microscopy (TEM) (JEOL, JEM$1200 \mathrm{EX}$ II). At least 500 polymer particles were measured for each latex sample. Emulsified monomer droplets in the latex sample were counted and recorded by a video-enhanced optical microscope (Olympus, $\mathrm{BH}-2$ ).

\section{RESULTS AND DISCUSSION}

Figure 1 shows monomer conversion $(X)$ vs. time $(t)$ profiles for the ST emulsion polymerizations carried out at $T=50-80^{\circ} \mathrm{C}$. Polymerization at 60,70 , or $80^{\circ} \mathrm{C}$ was carried out twice to check reproducibility. The reproducibility of $X v s$. $t$ data is reasonably good, as shown in Figure 1. Styrene can polymerize in the absence of initiator when $T$ is above $60^{\circ} \mathrm{C}$. To study such thermal polymerization, ST emulsion polymerization without SPS was carried out at $80^{\circ} \mathrm{C}$. After $3 \mathrm{~h}$ (emulsification

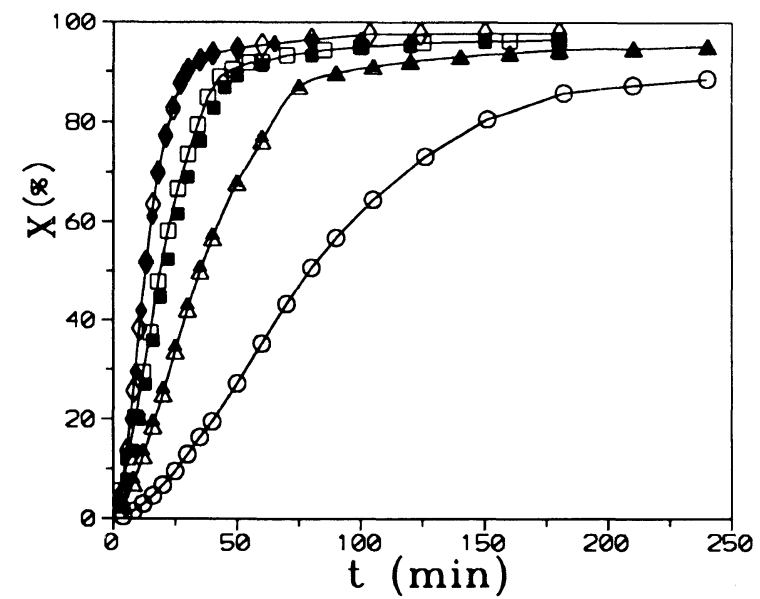

Figure 1. Monomer conversion as a function of reaction time. Polymerization temperature: $(\bigcirc) 50 ;(\triangle, \Delta) 60 ;(\square, \square) 70 ;(\diamond, \diamond)$ $80^{\circ} \mathrm{C}$.

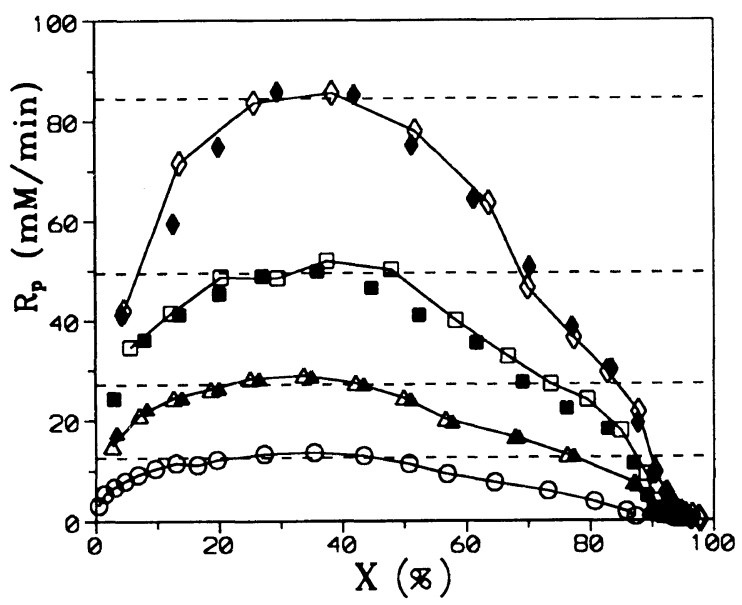

Figure 2. Polymerization rate as a function of monomer conversion Polymerization temperature: $(\bigcirc) 50 ;(\Delta, \Delta) 60 ;(\square, \square) 70 ;(\diamond, \diamond)$ $80^{\circ} \mathrm{C}$.

time $=2 \mathrm{~h}$ and $t=60 \mathrm{~min}$, see the EXPERIMENTAL section), the monomer conversion achieved was only $0.8 \%$. For polymerization initiated by $1.4 \times 10^{-3} \mathrm{M}$ SPS at $80^{\circ} \mathrm{C}$, conversion at $t=60 \mathrm{~min}$ was $95 \%$ (see the $\diamond$ and data points in Figure 1). This suggests that the influence of thermal polymerization on the kinetics of the ST emulsion polymerizations at $T=50-80^{\circ} \mathrm{C}$ is negligible. The corresponding polymerization rate $\left(R_{\mathrm{p}}=\right.$ $\left.[\mathrm{M}]_{0} \mathrm{~d} X / \mathrm{d} t\right)$ data are shown in Figure 2, in which $[\mathrm{M}]_{0}$ is the initial monomer concentration in the reaction system. The numerical formulation $\mathrm{d} X_{\mathrm{i}} / \mathrm{d} t_{\mathrm{i}}=\left(X_{i+1}-\right.$ $\left.X_{i-1}\right) /\left(t_{i+1}-t_{i-1}\right)$ was used to compute the slope of $X$ vs. $t$ curve. The dashed line in the plot indicates the magnitude of $R_{\mathrm{p}}$ in the constant polymerization rate region, generally defined as the Smith-Ewart Interval II. At constant $T, R_{\mathrm{p}}$ first increases rapidly with $X$ as the particle nuclei (reaction loci) continue to form, presumably via the micellar nucleation mechanism, ${ }^{1-3}$ in the reaction mixture $(X<20 \%$, Smith-Ewart Interval I). $R_{\mathrm{p}}$ remains relatively constant during the SmithEwart Interval II $(20 \%<X<50 \%)$. This is followed by significant reduction in $R_{\mathrm{p}}$ toward the end of polymerization as a result of the continuous decrease in $[\mathrm{M}]_{\mathrm{p}}(X>50 \%$, Smith-Ewart Interval III). As expected, 


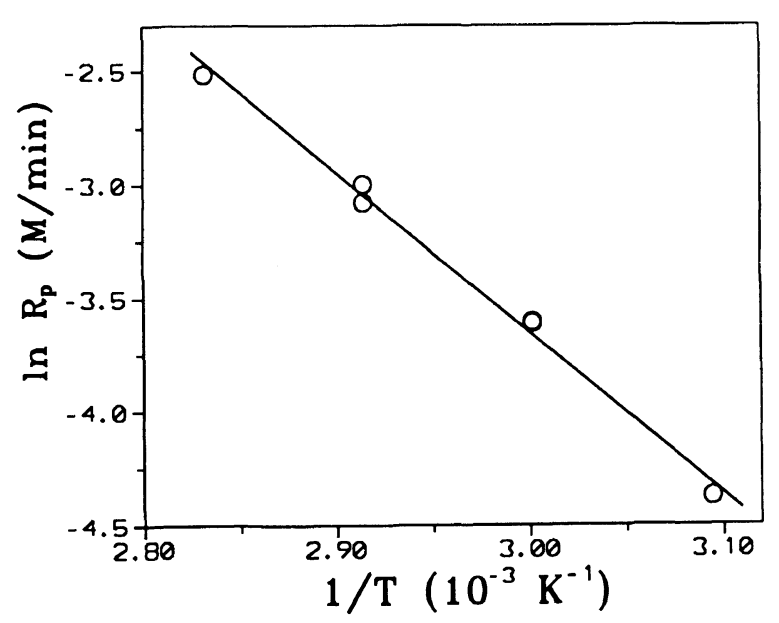

Figure 3. Arrhenius plot of the polymerization rate determined in the Smith-Ewart Interval II vs. reciprocal of temperature.

$R_{\mathrm{p}}$ increases rapidly with $T$ (see Figure 2). Figure 3 shows Arrhenius plots of $\ln R_{\mathrm{p}} v s .1 / T$ data and the least-squares best-fitted relationship is $R_{\mathrm{p}}=4.13 \times 10^{7} \exp (-3088 / T)$, in which $R_{\mathrm{p}}$ data were taken during Interval II and $R_{\mathrm{p}}$ and $T$ are in $\mathrm{mol} \mathrm{L}^{-1}$-min and $K$, respectively. The overall activation energy for the polymerization rate obtained from the least-squares best-fitted slope of the $\ln R_{\mathrm{p}} v s$. $1 / T$ data is $58.8 \mathrm{~kJ} \mathrm{~mol}^{-1}$. It is interesting to note in Figure 2 that the conversion range with constant $R_{\mathrm{p}}$ becomes narrower as $T$ increases from 50 to $80^{\circ} \mathrm{C}$.

Dried polymer particle size distribution (PSD) data as a function of $X$ for the polymerizations performed at $T=50-80^{\circ} \mathrm{C}$ are illustrated in Figure 4. A general feature of the PSD profile is the primary peak with a long tail comprising smaller particles. This tail becomes more pronounced at higher monomer conversion. The major peak is attributed to latex particles originating from micellar nucleation. ${ }^{1-3}$ In contrast, the long tail is probably caused by a homogeneous nucleation mechanism. ${ }^{13-15}$ According to this mechanism, particle nuclei are generated by precipitation of the oligomeric radicals with a critical chain length out of the aqueous phase. The new particles may continue to grow by capture of oligomeric radicals, monomer and surfactant from the aqueous phase. Limited flocculation among these particle nuclei may take place in the polymerization system. This flocculation greatly reduces total particle surface area, increases particle surface charge density and, thereby, enhances the colloidal stability of the aggregated particles (termed as the primary particles). The surfactant species required to stabilize the resultant primary particles may come from those dissolved in water, those released from disbanded micelles or those adsorbed on the monomer droplet surfaces. Polydispersity index $\left(d_{w} / d_{n}\right)$ data as a function of $X$ for the polymerizations at $T=50-80^{\circ} \mathrm{C}$ fall within the range of $1.04-1.14$. Parameters $d_{w}$ and $d_{n}$ are weight-average and number-average diameters of the dried polymer particles, respectively. The $d_{w} / d_{n}$ data for the polymerization carried out at a certain $T$ are very scattered and no apparent correlation between $d_{w} / d_{n}$ and $X$ can be identified (data not shown). The influence of $T$ on the PSD data is not straightforward. The reason for the scattered $d_{w} / d_{n}$ data is not clear, but may be closely related to the complicated particle nucleation,

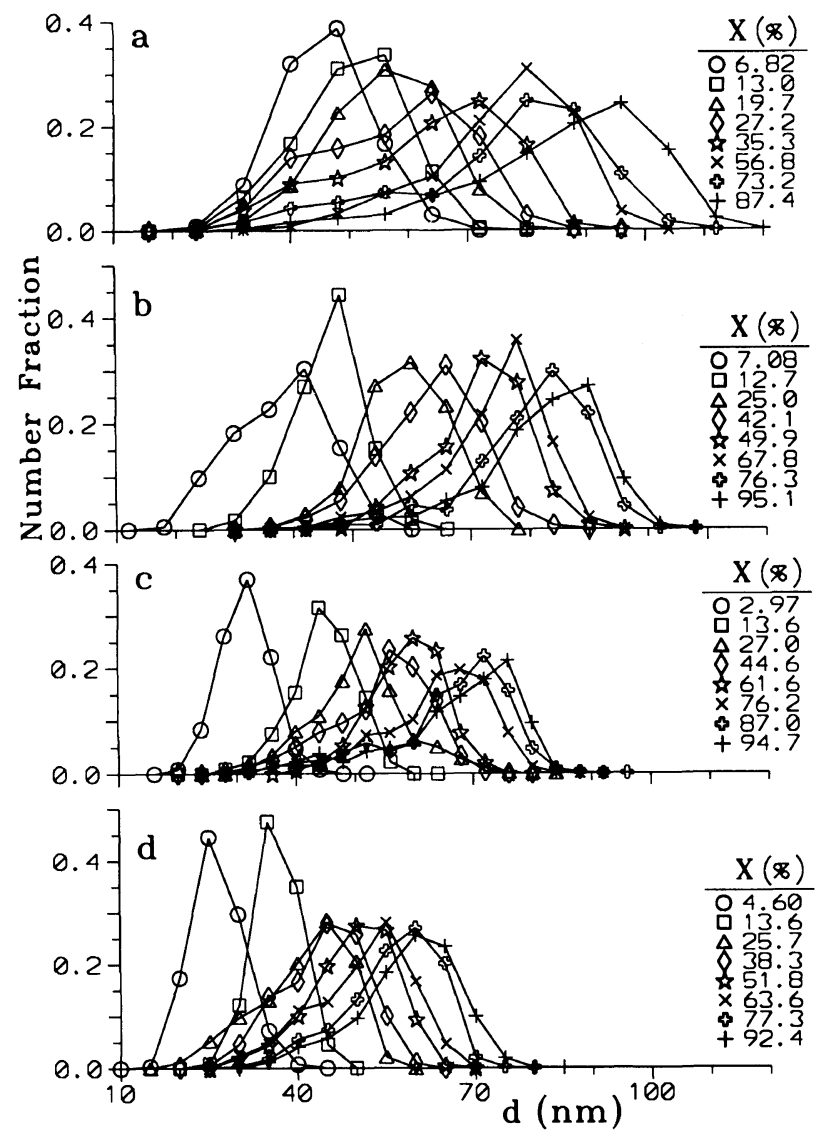

Figure 4. Dried latex particle size distribution as a function of monomer conversion. Polymerization temperature: (a) 50; (b) 60; (c) 70 ; (d) $80^{\circ} \mathrm{C}$.

growth and/or flocculation mechanisms. PSD of the latex product prepared at $T=50-80^{\circ} \mathrm{C}$ is quite broad, as shown by the representative TEM photographs in Figure 5 . It is interesting to note that a small population of tiny polymer particles is observed for each latex sample. Figure 6 shows (a) weight-average latex particle diameter $\left(d_{w}\right)$ and (b) the number of latex particles per liter water $\left(N_{\mathrm{p}}\right)$ as a function of $X$ for polymerization at $T=50-80^{\circ} \mathrm{C}$. At constant $T, d_{w}$ first increases rapidly from 0 to $30-50 \mathrm{~nm}$ during early polymerization $(X=0-10 \%)$, as shown in Figure 6a. Subsequently, $d_{w}$ increases gradually with polymerization. At constant $X$, $d_{w}$ decreases significantly with increasing $T$. Polymerization can be roughly divided into three stages according to $N_{\mathrm{p}}$ vs. $X$ data shown in Figure 6b. First, $N_{\mathfrak{p}}$ increases rapidly from 0 to $2 \times 10^{17}-6 \times 10^{17} \mathrm{~L}^{-1}$ during early polymerization $(X=0-5 \%) . N_{\mathrm{p}}$ increases steadily at $X$ from $5 \%$ to $30 \%$ for polymerization at 50 or $60^{\circ} \mathrm{C}$. For polymerization at higher temperature $\left(70\right.$ or $\left.80^{\circ} \mathrm{C}\right), N_{\mathrm{p}}$ increases gradually at $X$ from $5 \%$ to $60 \%$. Subsequently, $N_{\mathrm{p}}$ remains relatively constant toward the end of polymerization because total particle surface area becomes large enough to capture particle nuclei produced in the aqueous phase. Such a $N_{\mathrm{p}} v s . X$ profile suggests that most latex particles are produced during early polymerization (Smith-Ewart Interval I), presumably via the micellar nucleation mechanism. At constant $X, N_{\mathrm{p}}$ increases with $T$ (see Figure $6 \mathrm{~b}$ ). Particle nuclei would thus appear continuously generated in polymerization. Such secondary particle nucleation is most likely caused 

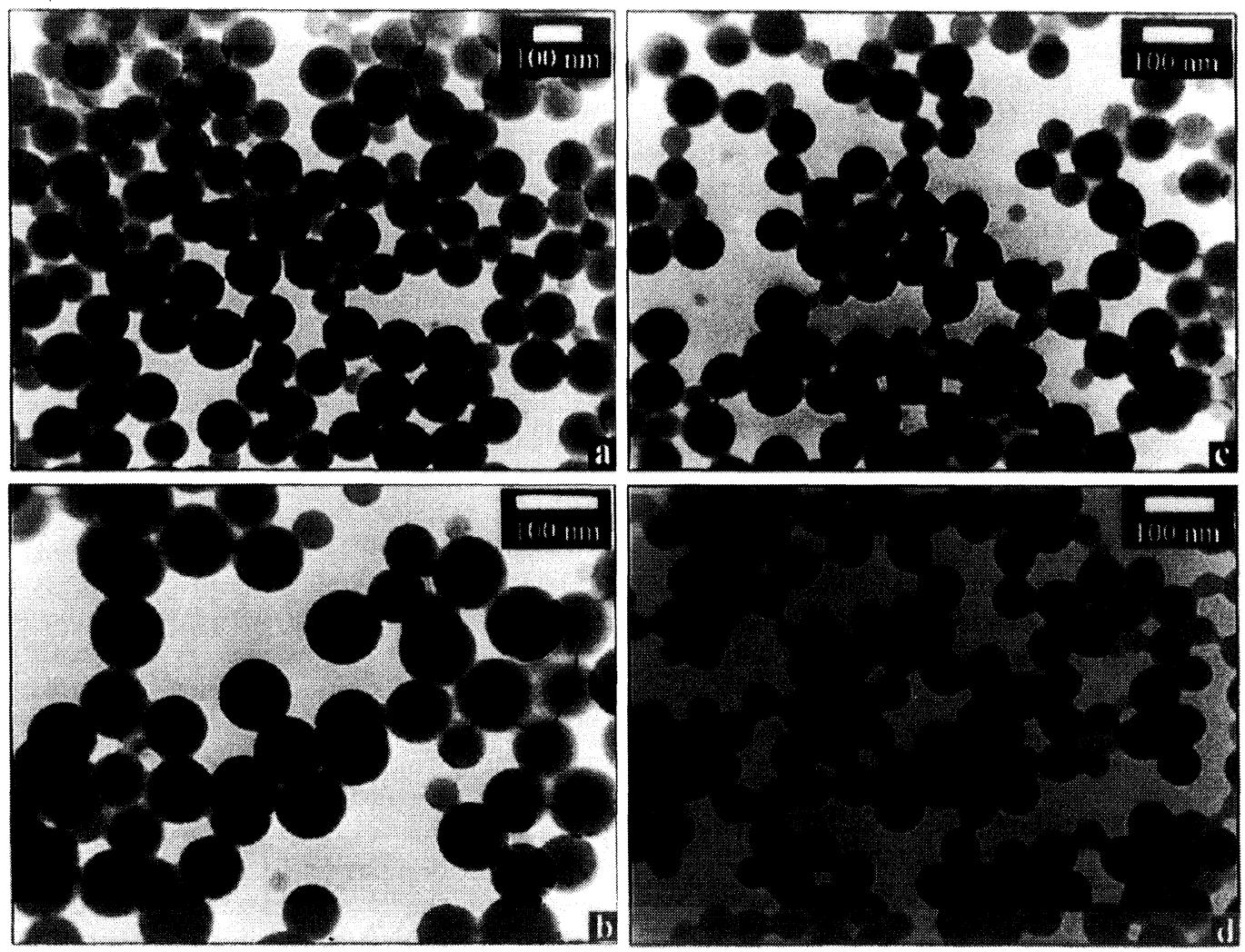

Figure 5. Representative TEM photographs for latex products. Polymerization temperature: (a) $T=50^{\circ} \mathrm{C}, X=89 \%, d_{\mathrm{w}}=85 \mathrm{~nm}$; (b) $T=60^{\circ} \mathrm{C}, X=90 \%, d_{\mathrm{w}}=78 \mathrm{~nm}$; (c) $T=70^{\circ} \mathrm{C}, X=91 \%, d_{\mathrm{w}}=65 \mathrm{~nm}$; (d) $T=80^{\circ} \mathrm{C}, X=90 \%, d_{\mathrm{w}}=58 \mathrm{~nm}$.
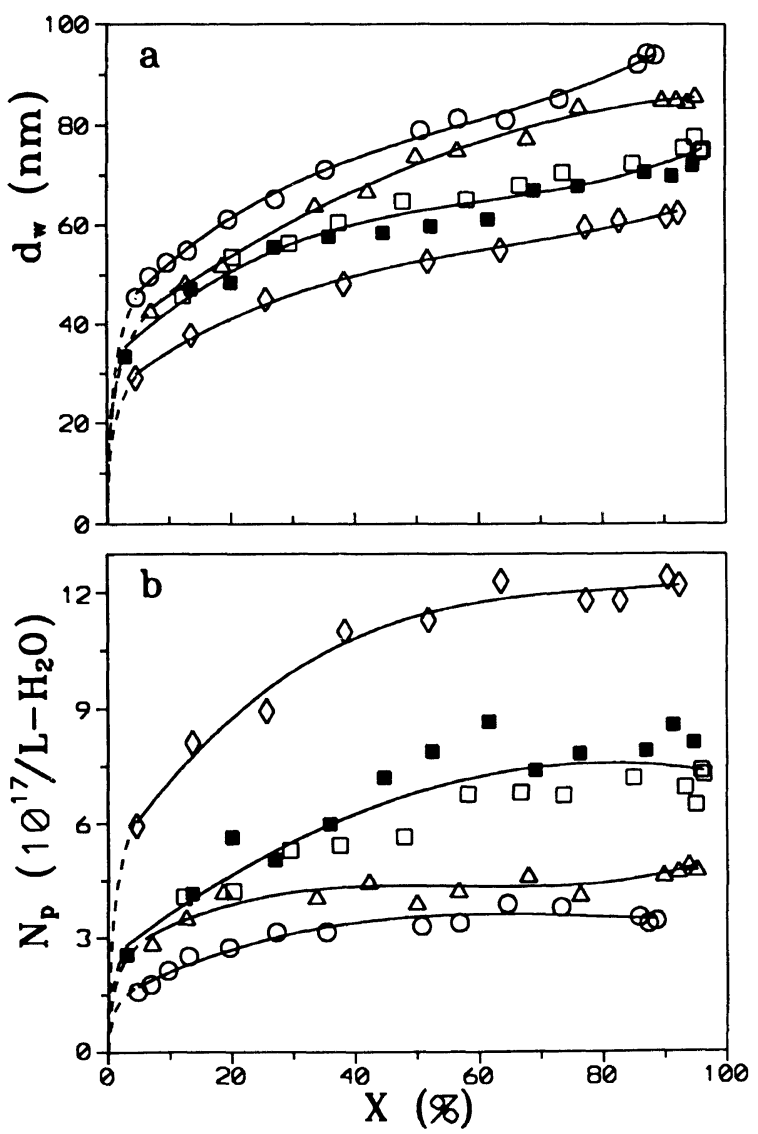

Figure 6. (a) Weight-average diameter of dried latex particles and (b) number of latex particles per liter water as a function of monomer conversion. Polymerization temperature: $(\bigcirc) 50 ;(\triangle) 60 ;(\square, \square) 70$; $(\diamond) 80^{\circ} \mathrm{C}$. by homogeneous nucleation ${ }^{13-15}$ since monomer-swollen micelles are expected to be consumed rapidly during early polymerization. The extent of secondary particle nucleation seems to increase with $T$, because the generation rate of the initiator radicals in water increases with $T$. Therefore, at constant ionic strength (note that both $[\mathrm{S}]$ and $[\mathrm{I}]$ were kept constant), the probability of successfully nucleating particle embryos in the continuous phase is greatly enhanced when polymerization is carried out at higher temperature. Unlike the latex sample with uniform particle size, the quite broad particle size distribution data obtained from latex samples taken during polymerization inevitably lead to uncertainty in the number of latex particles nucleated. Thus, $N_{\mathrm{p}}, \bar{n}$, and other kinetic parameters (see below) reported in this work at the best represent average values. The polymerization kinetics described hereafter should thus be regarded qualitatively.

With the knowledge of $R_{\mathrm{p}}, k_{\mathrm{p}},[\mathrm{M}]_{\mathrm{p}}$, and $N_{\mathrm{p}}$, eq 2 can be used to calculate the average number of free radicals per particle $(\bar{n})$ for ST emulsion polymerization. The $k_{\mathrm{p}}$ and $[\mathrm{M}]_{\mathrm{p}}$ data taken from ref 16 and 4 , respectively, can be found in Table I. The $\bar{n} v s$. $X$ data for polymerization at $T=50-80^{\circ} \mathrm{C}$ are shown in Figure 7 . The dashed line in the plot represents the condition corresponding to the Smith-Ewart Case 2 kinetics $(\bar{n}=0.5)$. At constant $T, \bar{n}$ does not change very much at constant polymerization rate. The $\bar{n}$ data (an average of all data points within the constant polymerization rate region) for polymerization at $T=50-80^{\circ} \mathrm{C}$ are $0.497,0.463,0.375$, and 0.255 , respectively (see Table II). Thus, at constant polymerization rate, the reaction system operated at 50 or $60^{\circ} \mathrm{C}$ follows the Smith-Ewart Case 2 kinetics reasonably well. 
The reaction system with $T>60^{\circ} \mathrm{C}$ deviates from SmithEwart Case 2 kinetics. In this case, the reaction system can be described by Smith-Ewart Case 1 kinetics $(\bar{n}<0.5)$. This is due to the fact that $d_{w}$ decreases and $N_{\mathrm{p}}$ increases at $T$ from 50 to $80^{\circ} \mathrm{C}$ (see Figure 6). The decomposition rate of initiator may thus not be sufficient to adequately supply radicals for the very large population of latex particles. The population of inactive particles containing no radicals in the reaction system becomes larger, thereby leading to reduction in $\bar{n}$. Another contributing factor is increased desorption of radicals out of the latex particles with $T$. The desorption mechanism involves transfer of activity of a macroradical to monomer, followed by diffusion of the mobile monomeric radical to the particle surface. The relatively water-soluble species may cross the interface into water. Finally, the radical exit is complete by diffusion of the monomeric radical to the bulk aqueous phase. The desorbed radical may be reabsorbed into another particle or terminated with an

Table I. Kinetic parameters for emulsion polymerization of styrene at various temperatures

\begin{tabular}{ccc}
\hline Parameter & Numerical value & Reference \\
\hline$[\mathrm{M}]_{\mathrm{p}}$ & $5.2 \mathrm{M}$ & 4 \\
$\rho_{\mathrm{m}}$ & $0.909 \mathrm{~g} \mathrm{ml}^{-1}$ & 23 \\
$f$ & 1 & \\
$k_{\mathrm{d}}$ & $9.083 \times 10^{17} \mathrm{exp}(-17885.6 / T) \mathrm{s}^{-1 \mathrm{a}, \mathrm{b}}$ & 16 \\
$k_{\mathrm{p}}$ & $1.052 \times 10^{9} \exp (-5052.3 / T) \mathrm{L} \mathrm{mol}^{-1} \mathrm{~s}^{-1}$ a.b & 16 \\
$k_{\mathrm{t}}$ & $8.2 \times 10^{9} \exp (-1747 / T) \mathrm{L} \mathrm{mol}^{-1} \mathrm{~s}^{-1 \mathrm{a}}$ & 24 \\
$Y$ & 0 & \\
$a^{\prime}$ & 1300 & 35 \\
$D_{\mathrm{w}} / D_{\mathrm{p}}$ & 10 & 33 \\
\hline
\end{tabular}

${ }^{\text {a }} T$ in $K$. ${ }^{\text {b }}$ Obtained by fitting the data with the Arrhenius equation.

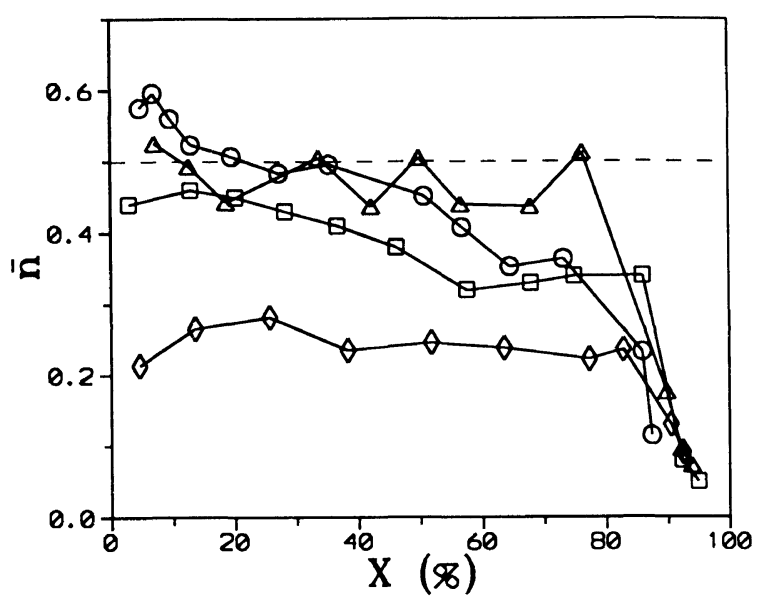

Figure 7. Average number of free radicals per particle as a function of monomer conversion. Polymerization temperature: $(\bigcirc) 50 ;(\triangle) 60$; (口) 70 ; $\diamond) 80^{\circ} \mathrm{C}$. oligomeric radical in the aqueous phase. The smaller the latex particles, the larger is the probability for the monomeric radical generated by the chain transfer reaction to diffuse from the particle phase into the aqueous phase. The parameter $\bar{n}$ may thus decrease to a value much lower than 0.5 as $T$ is increased from 50 to $80^{\circ} \mathrm{C}$.

It is generally accepted that emulsified monomer droplets completely disappear immediately after the end of Smith-Ewart Interval II. Reported $X$ at which point the monomer droplets vanish out of the polymerization system range from $40 \%$ to $60 \% .^{1,6,17,18}$ Representative optical photographs showing images of monomer droplets in the latex samples taken during polymerization at $60^{\circ} \mathrm{C}$ are shown in Figure 8 . Both the number and size of the monomer droplets decrease as polymerization proceeds. In particular, monomer droplet size decreases significantly only after $35-50 \%$ of the monomer has been converted to polymer. A significant reduction in the number of monomer droplets is observed at $90 \%$ conversion. Beyond $95 \%$ conversion, eventually no monomer droplets can be found in the polymerization system. The experiments conducted at 50,70 , and $80^{\circ} \mathrm{C}$ show similar results. The question is then why a significant population of tiny monomer droplets are still present in the reaction system well beyond the SmithEwart Interval II. One possible explanation is that, in the course of polymerization, polymer (or oligomer) may form inside the monomer droplets. ${ }^{19}$ The presence of polymer in the monomer droplets may be caused by capture of radicals by the droplets or thermal polymerization inside the droplets. Just like the role of cosurfactant (e.g., cetyl alcohol) in the stabilization of miniemulsion, the hydrophobic polymer may severely retard the diffusion of monomer from the monomer droplets, through the continuous phase, and then into growing latex particles. Recently, Schork et al. ${ }^{20,21}$ used a polymeric hydrophobe to prepare kinetically stable miniemulsions and these results provide supporting evidence for the above postulation. This retarded monomer diffusion may also have an impact on the dynamic partition of monomer among the monomer droplet phase, aqueous phase and monomer-swollen polymer particle phase in conventional ST emulsion polymerization. Nevertheless, further research is required to verify this possibility and a related project is currently in progress in our laboratory.

The $\log N_{\mathrm{p}} v s . \log \rho_{\mathrm{i}}$ data for the polymerizations at $T=50-80^{\circ} \mathrm{C}$ are presented in Figure 9. The $N_{\mathrm{p}}$ data shown in this plot represent those determined at the end of polymerization. The values of $k_{\mathrm{d}}$ at various temperatures used to calculate $\rho_{\mathrm{i}}$ were taken from ref 16 and $f$ is assumed to be unity (see Table I). The

Table II. Theoretical analysis of the transport of free radicals at constant polymerization rate

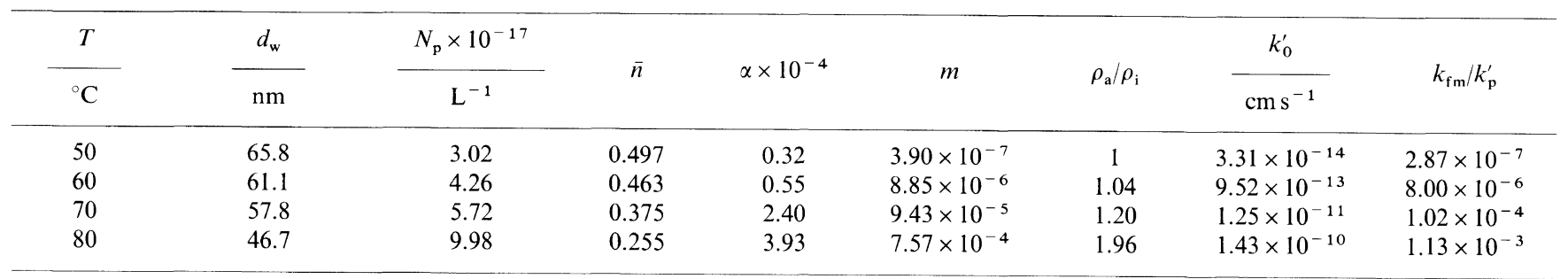



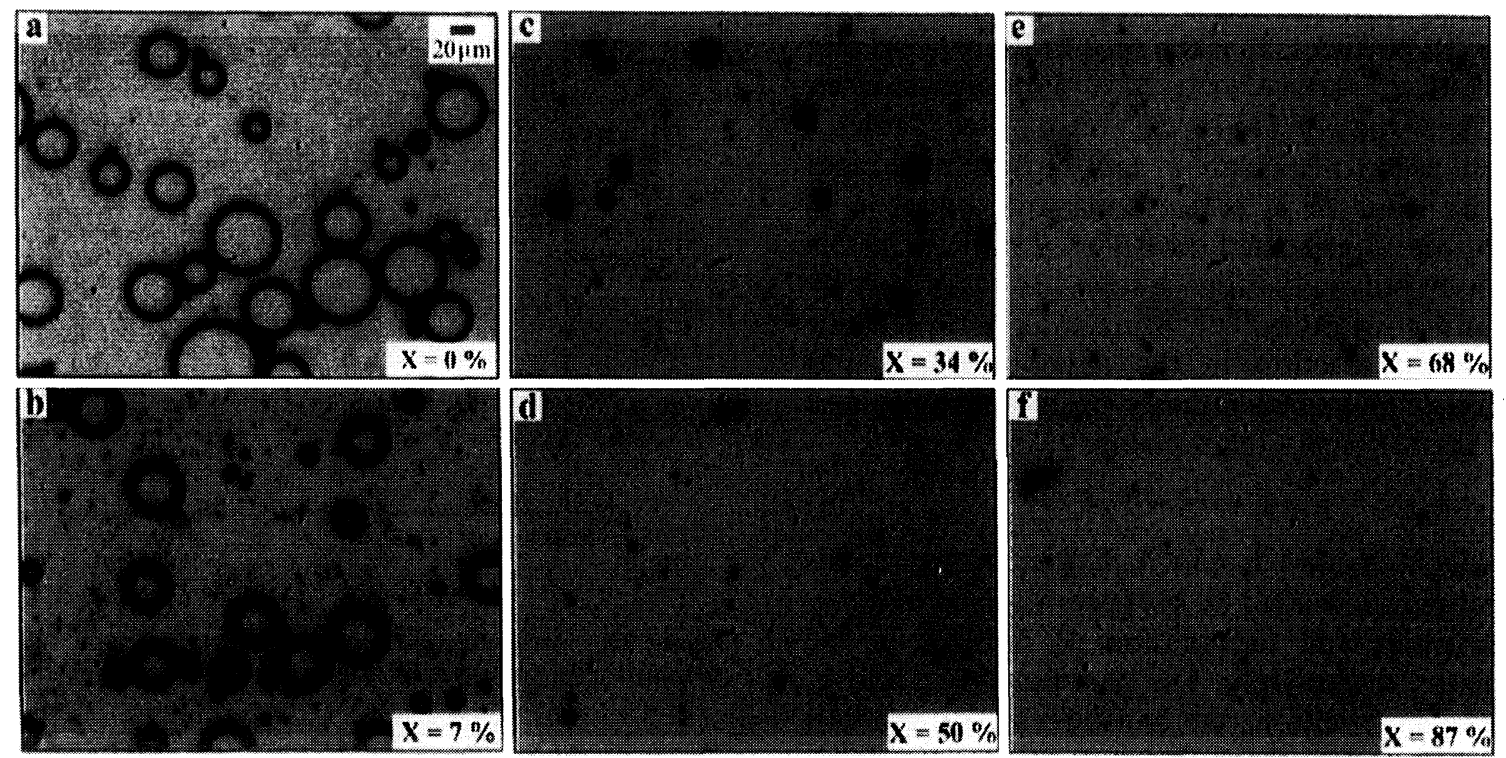

Figure 8. Representative optical photographs showing images of monomer droplets in the latex samples taken during polymerization at $60^{\circ} \mathrm{C}$. Monomer conversion: (a) $0 \%$; (b) $7 \%$; (c) $34 \%$; (d) $50 \%$; (e) $68 \%$; (f) $87 \%$.

relationship $N_{\mathrm{p}} \propto \rho_{\mathrm{i}}^{0.27}$ holds in this case and is not consistent with the Smith-Ewart Case 2 model. The discrepancy is probably due to the fact that: (1) generation of the particle nuclei does not cease beyond the Smith-Ewart Interval I due to the mixed mode of particle nucleation (micellar nucleation/homogeneous nucleation), (2) the range of the constant polymerization rate region (Smith-Ewart Interval II) becomes narrower at $T$ from 50 to $80^{\circ} \mathrm{C}$, (3) polymerization operated at higher temperature $\left(>60^{\circ} \mathrm{C}\right)$ does not obey the SmithEwart Case 2 kinetics $(\bar{n}=0.5)$, and (4) appreciable tiny monomer droplets remain in the reaction mixture beyond the Smith-Ewart Interval II. The experimental observations (1), (3), and (4) are in contradiction to the Harkins-Smith-Ewart theory. Chatterjee et al. ${ }^{7}$ suggest that, in the presence of SDS, the decomposition rate of the persulfate initiator might increase and SDS radicals derived from chain transfer of the initiator radicals to the surfactant species participate in the polymer reaction in the aqueous phase, in monomer-swollen micelles or growing particles. The degree of side reactions is expected to increases with $T$ because of enhanced initiator radical concentration with $T$. These side reactions may add extra complexity to particle formation. Recently, Chang et $a l^{22}$ used a 4-terminal cell and impedance spectrometer to determine the critical micelle concentration (CMC) of a similar ST/SDS/water macroemulsion at $25^{\circ} \mathrm{C}$. They found that the $\mathrm{CMC}$ of the macroemulsion is about $10 \%$ greater than that of the SDS aqueous solution $(6.7 \mathrm{mM})$. Increased CMC was attributed to adsorption of SDS molecules on the monomer droplet-water interfacial area. The recipe used in this work contains $18.2 \mathrm{mM}$ SDS and this level of surfactant assures the presence of abundant monomer-swollen micelles for particle nucleation in subsequent emulsion polymerization. The abundant supply of surfactant in the reaction system may be responsible for the continuous particle nucleation behavior. Immediately before polymerization, CMC and aggregation numbers of the monomer-swollen micelles for the ST/SDS/SPS/water macroemulsions at $T=50$

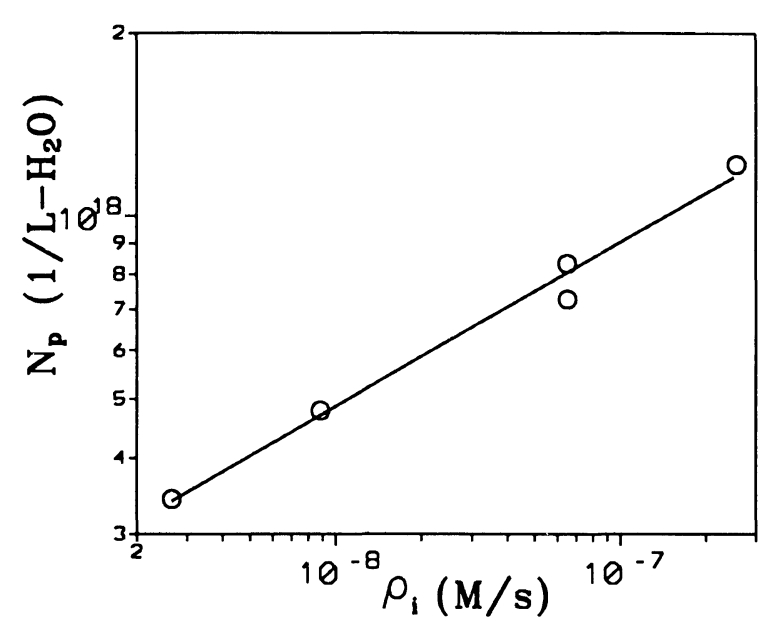

Figure 9. Number of latex particles per liter water as a function of production rate of initiator radicals in the aqueous phase.

$80^{\circ} \mathrm{C}$ are not known. The polymerization temperature may affect the concentration of monomer-swollen micelles produced and solubility of water-born oligomeric radicals (i.e., critical chain length of oligomeric radicals to start homogeneous nucleation). These factors may have impact on particle formation.

Finally, $d_{w}, N_{\mathrm{p}}$ and $\bar{n}$ determined within the constant polymerization rate region were used to study the transport of free radicals between the aqueous phase and the monomer-swollen polymer particle phase. O'Toole's approach $^{25}$ was used to calculate $\bar{n}$ at pseudo-steady state:

$$
n=a / 4\left[I_{m}(a) / I_{m-1}(a)\right]
$$

where $a=(8 \alpha)^{1 / 2}$ and $I_{m}$ and $I_{m-1}$ are the Bessel functions of the first kind of order $m$ and $m-1$, respectively. The kinetic parameters $\alpha=\rho_{\mathrm{a}} v_{\mathrm{p}} / k_{\mathrm{t}} N_{\mathrm{p}}$ and $m=k_{0} a_{\mathrm{p}} / k_{\mathrm{t}}$ are the dimensionless groups related to absorption of free radicals by latex particles and desorption of radicals out of the particles, respectively. The symbol $\rho_{\mathrm{a}}$ is the absorption rate of radicals by the particles, $v_{\mathrm{p}}$ is volume 
of monomer-swollen particles, $k_{\mathrm{t}}$ is the termination rate constant in the particles, $k_{0}$ is the specific desorption rate constant, and $a_{\mathrm{p}}$ surface area of a monomer-swollen particle. The values of $[\mathrm{M}]_{\mathrm{p}}, d_{w}$ and $\rho_{\mathrm{m}}$ (see Tables I and II) were used to calculate $v_{\mathrm{p}}$ and $a_{\mathrm{p}}$ by simple mass balance. The value of $\rho_{\mathrm{a}}$ is not necessarily equal to $\rho_{\mathrm{i}}$ due to the quite complicated transport of radicals in this heterogeneous polymerization system, as illustrated by Ugelstad and coworkers. ${ }^{26,27}$ They took into account the termination reaction in the aqueous phase and reabsorption of desorbed radicals by the particles and developed the following relationship:

$$
\alpha=\alpha^{\prime}+m \bar{n}-Y \alpha^{2}
$$

where $\alpha^{\prime}=\rho_{\mathrm{i}} v_{\mathrm{p}} / k_{\mathrm{t}} N_{\mathrm{p}}$ and $Y=2 N_{\mathrm{a}} k_{\mathrm{tw}} k_{\mathrm{t}} / k_{\mathrm{c}}^{2} v_{\mathrm{p}} N_{\mathrm{p}}$ are the dimensionless groups related to the generation of initiator radicals in water and termination of radicals in the aqueous phase, respectively. The parameters $k_{\mathrm{tw}}$ and $k_{\mathrm{c}}$ represent the termination rate constant in the aqueous phase and rate constant for the capture of radicals by the particles, respectively.

With the assumption that termination in the aqueous phase is not important (i.e., $Y=0$ ), $\alpha$ (or $\rho_{\mathrm{a}}$ ) and $m$ (or $k_{0}$ ) can be calculated as,

(1) Guess a value for $m$.

(2) Compute $\alpha$ according to eq 4 .

(3) Compute $\bar{n}$ by eq 3 .

(4) If calculated $\bar{n}$ is equal to the experimental value, accept the theoretical values of $\alpha$ and $m$. If not, then go back to step 1 .

(5) Compute $\rho_{\mathrm{a}}=\alpha k_{\mathrm{t}} N_{\mathrm{p}} / v_{\mathrm{p}}$ and $k_{0}=m k_{\mathrm{t}} / a_{\mathrm{p}}$.

The kinetic parameters necessary for calculations are obtained from the literature or estimated from the reaction conditions and are listed in Table I. The specific desorption rate constant $k_{0}\left(\mathrm{~cm} \mathrm{~s}^{-1}\right)$ is related to $k_{0}^{\prime}$ $\left(\mathrm{cm}^{2} \mathrm{~s}^{-1}\right)$ by the following equations: ${ }^{26-33}$

$$
\begin{gathered}
k_{0} a_{\mathrm{p}} / v_{\mathrm{p}}=k_{0}^{\prime} /\left[(\pi / 6)^{2 / 3} d_{\mathrm{p}}^{2}\right] \\
k_{0}^{\prime}=k_{\mathrm{fm}} / k_{\mathrm{p}}^{\prime}\left[12(\pi / 6)^{2 / 3} D_{\mathrm{w}} /\left(a^{\prime}+D_{\mathrm{w}} / D_{\mathrm{p}}\right)\right]
\end{gathered}
$$

where $d_{\mathrm{p}}$ is the diameter of a monomer-swollen particle, $k_{\mathrm{fm}}$ is the monomer chain transfer constant, $k_{p}^{\prime}$ is the reinitiation rate constant for monomeric radicals, $D_{\mathrm{w}}$ and $D_{\mathrm{p}}$ are the diffusion coefficients of the monomeric radicals in the water and particle phases, respectively, and $a^{\prime}$ is the partition coefficient for monomeric radicals between the particle phase and the aqueous phase. The parameter $k_{0}^{\prime}$ is independent of particle size and, therefore, it is reasonable to compare $k_{0}^{\prime}$ with the literature. The value of $t$ at the midpoint of the constant polymerization rate region was used in the calculation of $\rho_{\mathrm{i}}=2 f k_{\mathrm{d}}[\mathrm{I}](t=0)$ $\exp \left(-k_{\mathrm{d}} t\right)$. Such a choice does not have significant effect on theoretical analysis. For example, using the reaction time corresponding to either end of the constant polymerization rate region in the calculation of $\rho_{\mathrm{i}}$ only results in an error of at most $2 \%$ in $k_{0}^{\prime}$. The value of $D_{\mathrm{w}}$ for ST at $60^{\circ} \mathrm{C}$ is $2 \times 10^{-5} \mathrm{~cm}^{2} \mathrm{~s}^{-1} \cdot{ }^{33}$ If the temperature dependence of $D_{\mathrm{w}}$ follows the Eyring rate theory (i.e., $\left.D_{\mathrm{w}} \propto T\right),{ }^{34}$ then $D_{\mathrm{w}}$ at 50,70 , and $80^{\circ} \mathrm{C}$ can be estimated to be $1.94 \times 10^{-5}, 2.06 \times 10^{-5}$, and $2.12 \times 10^{-5} \mathrm{~cm}^{2} \mathrm{~s}^{-1}$, respectively. The values of $a^{\prime}$ and $D_{\mathrm{w}} / D_{\mathrm{p}}$ were taken from the literature and are included in Table I.

The results of theoretical analysis are summarized in

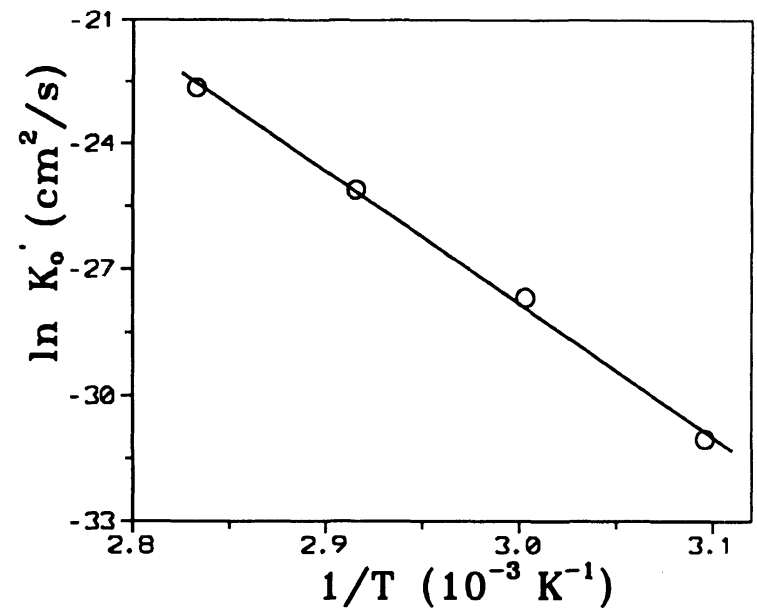

Figure 10. Arrhenius plots of the desorption rate constant (determined in the Smith-Ewart Interval II) vs. reciprocal of temperature.

Table II. The ratio $\rho_{\mathrm{a}} / \rho_{\mathrm{i}}$ first remains relatively constant at $c a .1$ when $T$ increases from 50 to $60^{\circ} \mathrm{C}$ (see Table II). This implies that diffusion of radicals from the particles into the aqueous phase does not have important influence on the kinetics of ST emulsion polymerization carried out at $T=50$ or $60^{\circ} \mathrm{C}$, as shown by the very small $k_{0}^{\prime}$ $\left(k_{0}^{\prime} \leq 9.52 \times 10^{-13}\right)$ in Table II. Thus, the absorption rate of radicals by the particles is very close to the production rate of radicals in water $\left(\rho_{\mathrm{a}} / \rho_{\mathrm{i}}=1\right)$. As a consequence, the reaction system follows the Smith-Ewart Case 2 kinetics $(\bar{n}=0.5)$ if $\rho_{\mathrm{i}}$ is large enough to make the particles active half the time in the course of polymerization. The ratio $\rho_{\mathrm{a}} / \rho_{\mathrm{i}}$ starts to increase from 1.04 to 1.96 as $T$ is increased from 60 to $80^{\circ} \mathrm{C}$. This is because the higher the polymerization temperature, the stronger the desorption of radicals out of the reaction loci. This is further supported by decreased $d_{w}$ (or $v_{\mathrm{p}}$ ) and rapidly increased $k_{0}^{\prime}$ at $T$ from 60 to $80^{\circ} \mathrm{C}$ (see Table II). That is, at higher $T$, monomeric radicals are present in particles with a smaller volume $\left(T \nearrow \Rightarrow v_{\mathbf{p}} \downarrow\right)$ and exhibit a larger desorption rate constant $\left(T \nearrow \Rightarrow k_{0}^{\prime} \nearrow\right)$. Both factors render these radicals more liable to escape from the particle phase. The population of radicals in the aqueous phase thus becomes larger than that originating from the decomposition of initiator only, leading to $\rho_{\mathrm{a}} / \rho_{\mathrm{i}}>1$. The resultant $k_{\mathrm{fm}} / k_{p}^{\prime}$ are included in Table II. The value of $k_{\mathrm{fm}} / k_{p}^{\prime}$ increases rapidly with $T$. In the calculation of $k_{\mathrm{fm}} / k_{p}^{\prime}, a^{\prime}$ and $D_{\mathrm{w}} / D_{\mathrm{p}}$ are assumed to be insensitive to change in $T$. Lee and Poehlein ${ }^{33}$ summarized $k_{0}^{\prime}$ for the ST emulsion polymerizations done by different research groups. The literature values of $k_{0}^{\prime}$ are in the range of $4.3 \times 10^{-13}-5.3 \times 10^{-13}$ and $3.9 \times 10^{-14}-4.8 \times 10^{-13}$ $\mathrm{cm}^{2} \mathrm{~s}^{-1}$ for polymerization at 50 and $60^{\circ} \mathrm{C}$, respectively. No $k_{0}^{\prime}$ data at 70 and $80^{\circ} \mathrm{C}$ are available in ref 33 . The values of $k_{0}^{\prime}$ at 50 and $60^{\circ} \mathrm{C}$ determined in this work are in reasonable agreement with the literature. The $\ln k_{0}^{\prime} v s$. $1 / T$ data are shown in Figure 10 and the least-squares best-fitted relationship is $k_{0}^{\prime}=1.64 \times 10^{29} \exp (-31698 /$ $T$ ), in which $k_{0}^{\prime}$ is in $\mathrm{cm}^{2} \mathrm{~s}^{-1}$ and $T$ is in $K$. The activation energy for the desorption process obtained from the least-squares best-fitted slope of the $\ln k_{0}^{\prime} v s .1 / T$ data is $264 \mathrm{~kJ} \mathrm{~mol}^{-1}$. Based on the kinetic data in this work, transport of radicals out of the growing particles does not show significant influence on polymerization kinetics 
and the reaction system can be adequately described by the Smith-Ewart Case 2 kinetics $(\bar{n}=0.5)$ when polymerization is performed at lower temperature $(T \leq$ $60^{\circ} \mathrm{C}$ ). The desorption becomes relatively important and results in lower $\bar{n}(\bar{n}<0.5)$ as $T$ is raised above $60^{\circ} \mathrm{C}$.

Acknowledgment. The financial support from National Science Council, Taiwan is gratefully acknowledged.

\section{REFERENCES}

1. W. D. Harkins, J. Am. Chem. Soc., 69; 1428 (1947)

2. W. V. Smith and R. W. Ewart, J. Chem. Phys., 16, 592 (1948).

3. W. V. Smith, J. Am. Chem. Soc., 70, 3695 (1948); ibid., 71, 4077 (1949).

4. E. Barthalome, H. Gerrens, R. Herbeck, and H. M. Weitz, Z. Elektrochem., 60, 334 (1956).

5. Z. Manyasek and A. Rezabek, J. Polym. Sci., 56, 47 (1962).

6. S. Omi, H. Sato, and H. Kubota, J. Chem. Eng. Japan, 2, 55 (1969).

7. S. P. Chatterjee, M. Banerjee, and R. S. Konar, J. Polym. Sci., Polym. Chem. Ed., 16, 1517 (1978)

8. C. S. Chern, S. Y. Lin, L. J. Chen, and S. C. Wu, Polymer, 38, 1977 (1997)

9. C. S. Chern, S. Y. Lin, S. C. Chang, J. Y. Lin, and Y. F. Lin, Polymer, 39, 2281 (1998).

10. B. V. Deryagnin and L. D. Landau, Acta Physicochim. USSR, 14, 633 (1941).

11. E. J. W. Verwey and J. Th. G. Overbeek, "Theory of the Stability of Lyophobic Colloids," Elsevier, New York, N.Y., 1943.

12. K. J. Mysels and L. H. Princen, J. Phys. Chem., 63, 1696 (1959).

13. C. P. Roe, Ind. Eng. Chem., 60, 20 (1968).

14. R. M. Fitch, M. B. Prenosil, and K. J. Sprick, J. Polym. Sci. C, 27, 95 (1969).

15. F. K. Hansen and J. Ugelstad, J. Polym. Sci., Polym. Chem. Ed., 16, 1953 (1978)

16. J. Brandrup and E. H. Immergut, "Polymer Handbook," 2nd ed,
Wiley-Interscience, New York, N.Y., 1975.

17. M. Harada, M. Nomura, H. Kojima, W. Eguchi, and S. Nagata, J. Appl. Polym. Sci., 16, 811 (1972).

18. W. Y. Chiu and C. C. Shih, J. Appl. Polym. Sci., 31, 2117 (1986)

19. I. Capek, Polymer Institute, Slovak Academy of Sciences, Slovak Republic, personal communication.

20. J. L. Reimers, A. H. P. Skelland, and F. J. Schork, Polymer Reaction Engineering, 3, 235 (1995)

21. J. Reimers and F. J. Schork, J. Appl. Polym. Sci., 59, 1833 (1996); ibid., 60, 251 (1996).

22. H. C. Chang, Y. Y. Lin, C. S. Chern, and S. Y. Lin, Langmuir, 14, 6632 (1998).

23. H. F. Mark, N. G. Gaylord, and N. Bikales, "Encyclopedia of Polymer Science and Technology," Interscience, New York, N.Y., 1964.

24. S. K. Soh and D. C. Sundberg, J. Polym. Sci., Polym. Chem. Ed., 20, 1345 (1982).

25. J. I. O’Toole, J. Appl. Polym. Sci., 9, 1291 (1965).

26. J. Ugelstad, P. C. Mork, and J. O. Aasen, J. Polym. Sci. A-1, 5, 2281 (1967).

27. J. Ugelstad and P. C. Mork, Br. Polym. J., 2, 31 (1970).

28. J. Ugelstad, P. C. Mork, D. Dahl, and P. Rangnes, J. Polym. Sci. Part C, 27, 49 (1969)

29. M. Harada, M. Nomura, W. Eguchi, and S. Nagata, J. Chem. Eng. Japan, 4, 54 (1971).

30. M. Nomura, M. Harada, K. Nakagawara, W. Eguchi, and S. Nagata, J. Chem. Eng. Japan, 4, 160 (1971).

31. M. Nomura, M. Harada, W. Eguchi, and S. Nagata, in "Emulsion Polymerization," I. Piirma and J. L. Gardon, Ed., ACS Symposium Series 24, American Chemical Society, Washington, D.C., 1970.

32. H. C. Lee, Ph.D. Dissertation, School of Chemical Engineering, Georgia Institute of Technology, Atlanta, GA, 1985.

33. H. C. Lee and G. W. Poehlein, Polym. Process Eng., 5, 37 (1987)

34. R. B. Bird, W. E. Stewart, and E. N. Lightfoot, "Transport Phenomena," Wiley, New York, N.Y., 1960.

35. M. Nomura, K. Yammota, I. Horie, and K. Fujita, J. Appl. Polym. Sci., 27, 2483 (1982). 